\title{
Natural Product Hybrids
}

\author{
Karl Gademann*
}

\begin{abstract}
This review highlights several classes of natural product hybrids. First, structural hybrids emanating from different biosynthetic pathways exemplified by the polyketide peptide alkaloid anachelin are discussed. Another class is the so-called functional hybrids, where different functions of natural products are hybridized. The conjugate between nostocarboline and the well-known antibacterial agent ciprofloxacin displays a very broad spectrum of activity combining photosynthesis and gyrase-inhibiting activity. Last, higher order hybrids are discussed. We present the ternary hybrid of titanium dioxide, the anachelin chromophore and poly-ethyleneglycol (PEG). This chimera allows for the functionalization of $\mathrm{TiO}_{2}$ surfaces to render those protein resistant. This article thus shows how natural products can be hybridized to generate compounds with unprecedented bioactivity thus enabling the development of novel drugs and advanced materials.
\end{abstract}

Keywords: Advanced materials · Antibacterial agents · Drug discovery · Natural products · Synthesis

\section{Introduction}

Natural products, i.e. chemical entities produced by living organisms such as bacteria, fungi and plants, have historically provided important molecular solutions to medical problems [1]. While extracts of plants have been traditionally used, the improvement of analytical techniques has facilitated the isolation and characterization of the active principles, the so-called natural products. Improved understanding of the mode of action of such compounds combined with the synthesis of derivatives enables the establishment of structure-activity relationships and thus the generation of more active, synthetic derivatives. This general strategy remains the most promising way to generate potent and selective biologically active compounds [2]. These statements are supported by analyses of new chemical entities introduced on the market in the period 1981 to 2002 [3]. Among these compounds around $40 \%$ are of natural origin or synthetic derivatives thereof. These numbers underline the tremendous importance of natural products in drug discovery.

The evolutionary wisdom enshrined in natural products can be unlocked by syn-

\footnotetext{
${ }^{*}$ Correspondence: Prof. Dr. K. Gademann Ecole Polytechnique Fédérale de Lausanne Institut des sciences et ingénierie chimiques EPFL-SB-ISIC-LSYNC

$\mathrm{CH}-1015$ Lausanne

E-Mail: karl.gademann@epfl.ch
}

thetic organic chemistry. The goal of this highlight article is to demonstrate that the biological functions of natural products can be expanded, combined and leveraged by so-called natural product hybrids (sometimes also referred to as chimeras or conjugates). This strategy is not limited to natural products themselves, as also synthetic compounds, from small molecules to polymers, can be hybridized to generate compounds with unprecedented functions. This highlight article will focus on our own work, as excellent general reviews on this subject have been published [4].

Analysis of natural products reveals an interesting pattern as many of these compounds are composed of substructures originating either from different biosynthetic pathways or containing several biological functions. These fragments are hybridized by nature to generate naturally occurring conjugates. Such hybrids include the wellknown glycosylated steroids, glycosylated or lipidated peptides, or polyketide alkaloids. In lipidated peptides, the lipid part is responsible for anchoring these compounds in biomembranes and the peptide part can exert the main biological function [5].

In this highlight article, we will discuss three classes of natural product hybrids relevant to our own work. The first example is a hybrid of different biosynthetic pathways (structural hybrid). The second part will demonstrate the hybridization of functions thus broadening the biological activity (functional hybrid). The last example will show how this concept can be expanded to generate ternary conjugates (higher order hybrids).

\section{Anachelin - A Structural Hybrid}

Siderophores are small molecules secreted by microorganisms to bind and sequester iron [6]. Our initial interest in these compounds originated from the historical evolution of earth's atmosphere. Initially, the earth was thought to contain a reductive atmosphere in which the early life forms flourished [7]. Their need for iron was supplemented by readily available and soluble Fe(II) salts [7]. Among these ancient organisms were also cyanobacteria (blue-green algae), with fossils dating their initial presence 3.5 billions years ago [8]. These organisms probably also developed and introduced photosynthesis on this planet and were thereby directly responsible for the release of oxygen [8]. Such a very successful photoautotrophic strategy led however to a change in earth's atmosphere as the reductive environment slowly changed into an oxidative one, as it is to this day. This change had dramatic consequences for many life forms present at that time as oxygen is both a potent oxidant and cell poison. Life forms surviving this disaster were soon faced with another challenge: Oxidation of the soluble Fe(II) salts to the corresponding virtually insoluble $\mathrm{Fe}$ (III), species of which the dominant iron oxide hydrates are in general not soluble ("the world rusted") [7]. Iron acquisition thus became a challenge for every organism. Although cyanobacteria probably caused this atmospheric change a long time ago, there is little known so far how these organisms faced this challenge and in particular, about complex cyanobacterial siderophores [8-10]. 
In 2000, the first complex siderophore from the cyanobacterium Anabaena cylindrica CCAP 1403-2a was isolated and named anachelin (1), as a mixture of two compounds [11] (Fig. 1). Later, additional isomers were reported [12]. The historical postulate outlined above that cyanobacteria were among the first organisms to encounter iron scarcity and met this challenge by use of small molecule siderophores stimulated us to initiate a research program aimed at these questions. In addition, the structure of anachelin (1) is appealing to the chemist. At least three different biosynthetic pathways are combined: The polyketide fragment, the peptide part featuring a non-standard D-amino acid, and the alkaloid fragment. In addition, the origin of the salicylate part is unknown and two biosynthetic pathways are likely for its generation. Therefore, anachelin (1) can be considered a structural natural product hybrid based on fragments of different biogenetic origin. We have investigated the solution structure of anachelin [13], the possible biosynthesis [14] and the mode of action of 1 [15], and completed its total synthesis thereby establishing the configuration [16][17]. This research enabled also the venture into surface functionalization using these compounds, as discussed below.

\section{Functional Hybrids Based on Nostocarboline}

Cyanobacteria (often referred to as bluegreen algae) are unicellular prokaryotic organisms. These photoautotrophs are among the oldest life-forms still present today on Earth and survived by populating almost every available ecological niche, from ice to rocks, from the open ocean to the fur of polar bears. In particular in warm and humid or aquatic habitats, intense competition for nutrients and light characterizes the evolutionary pressure on the organisms. In addition and similar to plants, cyanobacteria can encounter pressure by grazing organisms. Therefore, it is not surprising that cyanobacteria developed sophisticated chemical defense strategies for survival. Many metabolites are thought to be produced in order to gain an evolutionary advantage over competing organisms [18]. One example, anachelin with respect to the sequestering of trace metals, is discussed above. Other examples include bioactive metabolites such as the cyanobacterial toxins.

Up to now over one hundred different bioactive compounds have been characterized from cyanobacteria. These neuro- and hepatotoxins include well-known compounds such as microcystin, saxitoxin and anatoxin [18]. The ecological role of these agents is suggested to be related to deterrent purposes with respect to grazing by insects. Another class of cyanobacterial

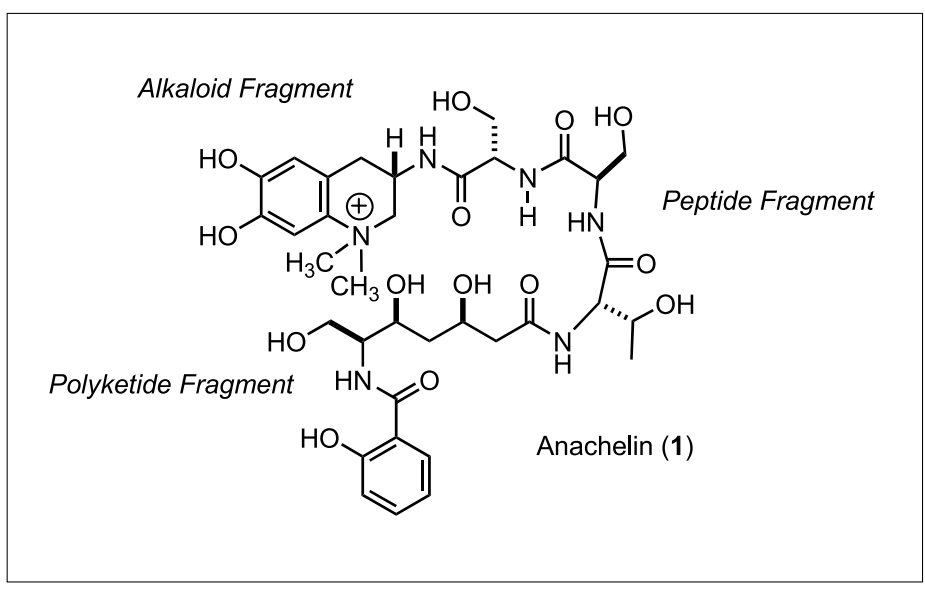

Fig. 1. Anachelin (1), a structural natural product hybrid featuring alkaloid, peptide and polyketide biosynthetic pathways

metabolites is directed towards competing photosynthetic species. For example, photosystem II inhibitors such as fischerellin A are thought to secure an evolutionary advantage by poisoning algae competing for the same resources [19].

While many bioactive compounds of cyanobacterial origin belong to the chemical class of modified peptides or mixed polyketide/peptide species [20], some alkaloids have also been isolated and characterized from cyanobacteria. In addition to the above-mentioned fischerellin, alkaloids with appealing molecular structure such as welwitindoline A (2) isolated from Hapalosiphon weilwitschi [21], and more simple fragments such as the bauerines (3a-c) [22] or norharmane (4) [23] and harmane [24] were isolated (Fig. 2).

In collaboration with the group of Prof. Dr. Friedrich Jüttner from the University of Zürich, we have characterized nostocarboline (5) from the toxic blue-green algae Nostoc 78-12A [25] (Fig. 3). This cyanobacterium, originally isolated from a wastewater lagoon in the USA, was reported to produce secondary metabolites with activities against photosynthetic organisms [26]. Bioassay-guided fractionation resulted in the identification of nostocarboline $(\mathbf{5})$, or 6-chloro-2-methyl-carbolinium [27]. Compound $\mathbf{5}$ is a so-called anhydronium base, a term coined by Sir Robert Robinson for bases [28], in which the proton and the pos-

itive charge (and thus the counterion) are spatially separated.

Nostocarboline (5) was found to be a potent inhibitor of butyrylcholinesterase, with an $\mathrm{IC}_{50}$ of $13.2 \mu \mathrm{M}$. This value is slightly lower that of galanthamine $(\mathbf{6}, 16.9$ $\mu \mathrm{M})$, which is on the market as a drug to treat Alzheimer's disease [29]. The related deschloro compound $\mathbf{7}$ has also a similar $\mathrm{IC}_{50}$ value. Interestingly, this compound is endogenous to humans, and its physiological role is unclear [29] (Fig. 3). Some reports raise the possibility that this compound is related to 1-methyl-4-phenylpyridinium (TPP), a known neurotoxin. Our data establishes that 2-methyl norhamane 7 is a butyrylcholinesterase inhibitor and this could imply such a physiological role for this orphan ligand [25]. As a side note, there are only few human alkaloids known such as harmane and norharmane. The physiological role of these remains the subject of intense scientific debate.

The producing strain Nostoc 78-12A was noted as anticyanobiotic by Flores and Wolk in the context of isolating and culturing this cyanobacterium [26]. Based on this precedent, we screened for phytotoxic properties of nostocarboline (5) to investigate whether this compound was responsible for the reported biological activity of the parent Nostoc strain. In fact, nostocarboline (5) was found to be very active against both prokaryotic and eukaryotic photoautotrophs,

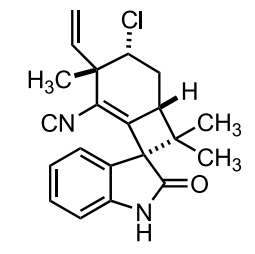

$\begin{array}{ll}\text { Welwitindoline A isonitrile (2) } & \begin{array}{l}\mathrm{R}=\mathrm{H} \text { : Bauerine A (3a) } \\ \mathrm{R}=\mathrm{Cl} \text { : Bauerine } \mathrm{B}(\mathbf{3 b})\end{array}\end{array}$

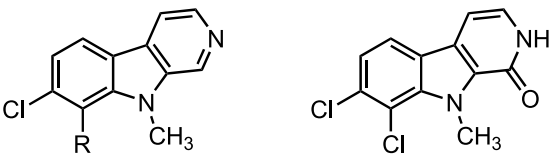

Bauerine C (3c)

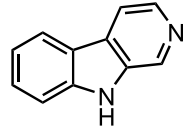

Norharmane (4) 


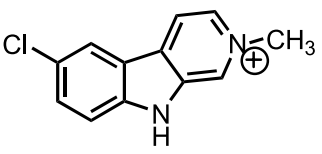

Nostocarboline (5)

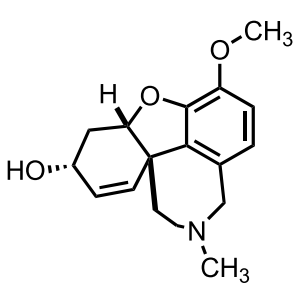

Galanthamine (6)
Deschloronostocarboline (7)

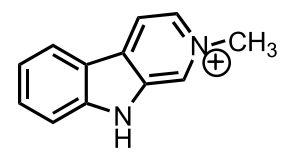

Clinical use against

Alzheimer's disease

Fig. 3. Nostocarboline (5), isolated from Nostoc 78-12A, is a potent inhibitor of butyrylcholinesterase of similar activity compared to galanthamine (6) and deschloronostocarboline (7). The latter compound was found in post-mortem human brain ('human alkaloid') [29].
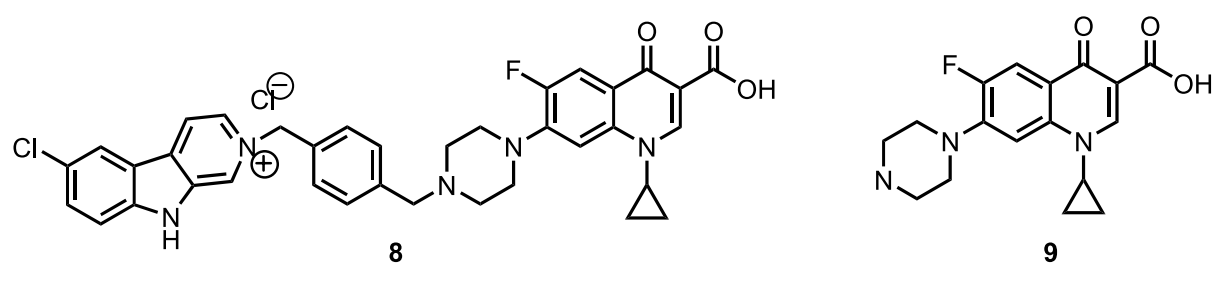

Fig. 4. The nostocarboline ciprofloxacin hybrid 8 and the parent antibacterial agent, ciprofloxacin (9).

with minimal inhibitory concentrations as low as $100 \mathrm{nM}$ [30]. This exceptionally low value in cell-based assays is remarkable, as the charged compound must first enter the cell and reach the target. Nostocarboline (5) was not active against non-photosynthetic pathogenic bacteria and fungi, which points to a target related to photosynthetic processes [31]. This is interesting, as the producing organism relies on photosynthesis as well. Autotoxicity of $\mathbf{5}$ against its producer was not found to be an issue (2 to 3 orders of magnitude of difference in MIC values), and exactly this difference could allow for evolutionary advantage against competing photoautotrophs [30].

Next, we asked whether the activity of 5 could be broadened to also include regular, non-photosynthetic bacteria. Such compound would prevent the growth of any prokaryotic and photoautotrophic organisms and could find interesting applications in biofilm control. The adhesion of bacteria, algae and higher organisms on the surfaces of ships, pipelines and platforms, as well as pipe systems for example in nuclear power plants constitutes a significant challenge. The problems are accentuated, as resistance towards $\mathrm{Cu}$-based antifouling paints is quickly developing, and traditional tinbased agents are increasingly being banned worldwide.

In order to broaden the activity of the phycotoxic agent nostocarboline, we prepared the hybrid 8 between nostocarboline (5) and ciprofloxacin (9), a very potent gyrase inhibitor and antibacterial agent. Both compounds are linked via an aryl linker to furnish the nostocarboline/ciprofloxacin hybrid 8 [30]. This compound has a unique profile of action retaining the phytotoxic properties of nostocarboline as well as the antibacterial activity of ciprofloxacin. MIC values as low as $0.7 \mu \mathrm{M}$ against $E$. coli as well as $1 \mu \mathrm{M}$ against the cyanobacterium $M$. aeruginosa have been determined. Similar quinolone chimeras have been reported to broaden antibacterial activity with concomitant decrease of resistance [32]

Chemically, hybridizing a putative photosynthesis inhibitor 5 and a gyrase inhibitor 9 (Fig. 4) without deterioration of potency is challenging as both targets must be reached by the same small molecule inhibitor. As this example shows, successful hybridiza- tion is only possible if structure-activity (SAR) relationships have been established. In this case, our work on nostocarboline (5) demonstrated that replacement of the 2-Me group by benzyl retained or slightly increased the activity [30]. Detailed SAR for ciprofloxacin (9) is well documented in the literature. This hybrid between the natural product nostocarboline and the synthetic (natural product derived) ciprofloxacin clearly demonstrates the power of the hybridization approach in combining and broadening bioactivity.

\section{Higher Order Hybrids}

As discussed above, the low bioavailability of iron has another consequence for the molecular mechanism of cyanobacterial iron acquisition. The prevalence of the dominant iron oxide hydrates requires that siderophores recognize and bind to the solid oxide surfaces. Siderophores were thus probably not only evolved to bind iron ions, but also to recognize and bind iron oxide surfaces. This hypothesis is supported by several studies demonstrating siderophore mineral interactions [33]. In particular there was one report of bacterial adhesion mediated by siderophores on titanium oxide surfaces [34]. Consequently, we postulated that siderophores are able to bind to metal oxides surfaces in general.

In order to test this hypothesis, the catecholate fragment of the siderophore anachelin (1) was hybridized to polyethylene glycol (PEG) and the resulting conjugate 10 (Fig. 5) evaluated for surface binding [35]. This potential success of this design is supported by several distinct structural features. First, the anachelin chromophore (the catecholate) could be biosynthetically related to dihydroxyphenylalaine (DOPA). This amino acid was found to be present in high content in socalled mussel adhesive proteins, which allow the mussels to bind to many surfaces

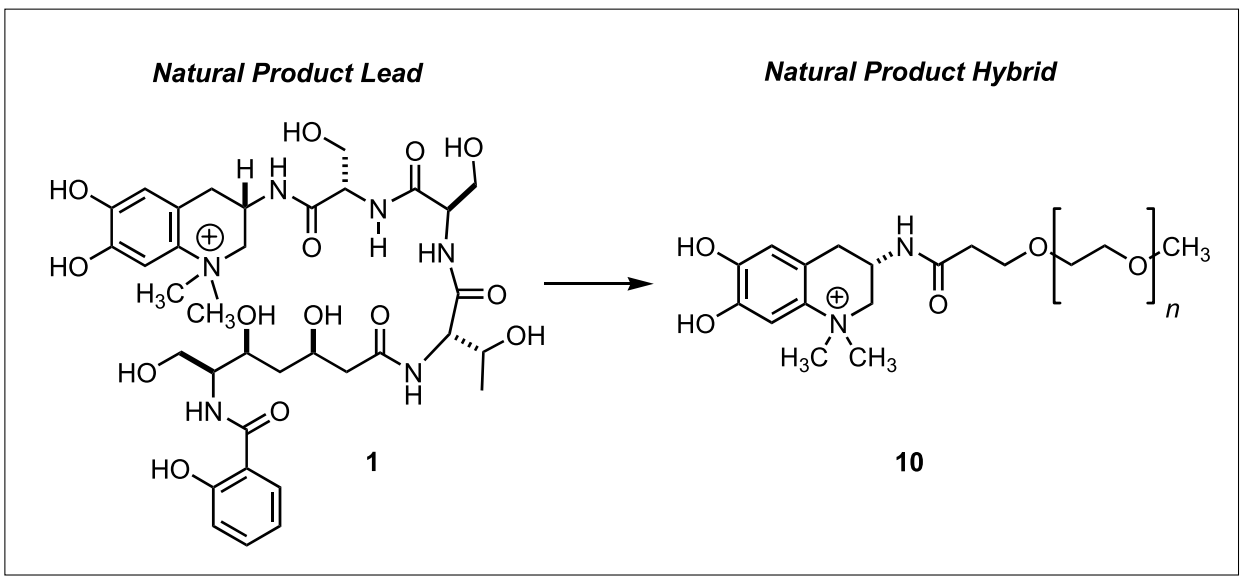

Fig. 5. The natural product lead, anachelin (1), allowed for the design of the natural product hybrid $\mathbf{1 0}$ consisting of the anachelin chromophore and poly-ethyleneglycol 
<smiles>CN(C)C[C@H](Cc1ccc(O)c(O)c1)NC(=O)OCc1ccccc1</smiles><smiles>COc1ccc([Te](=O)(c2ccc(OC)cc2)C2CC2)cc1</smiles>

$\mathrm{CH}_{2} \mathrm{Cl}_{2}$

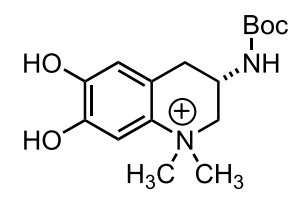

$12(64 \%)$

Scheme 1. Preparation of the anachelin chromophore

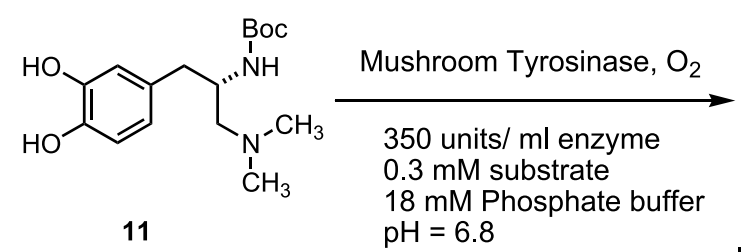<smiles>C[N+]1(C)C[C@@H](NC(=O)c2ccccc2)Cc2cc(O)c(O)cc21</smiles>

1. $\mathrm{HCl} /$ Dioxane 2. $\mathrm{mPEG}-\mathrm{OCH}_{2} \mathrm{CH}_{2} \mathrm{CO}_{2} \mathrm{Su}$

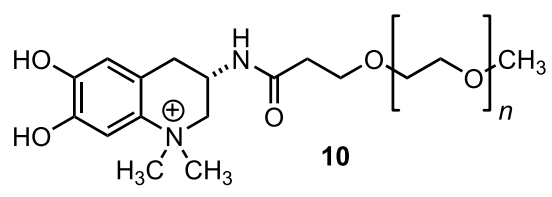

Scheme 2. Enzymatic preparation of the anachelin chromophore 12 and its conversion to the PEG conjugate 10

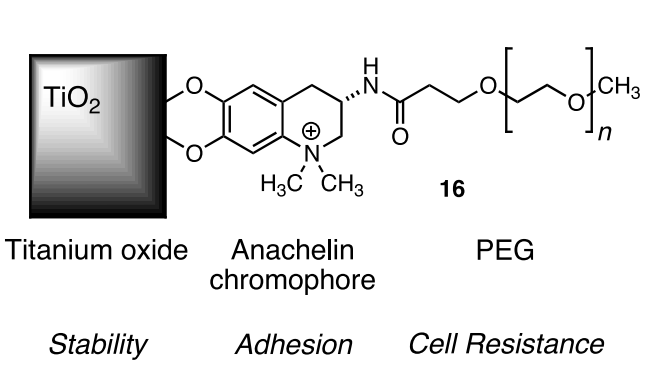

Polyethylene glycol Headgroup Surface

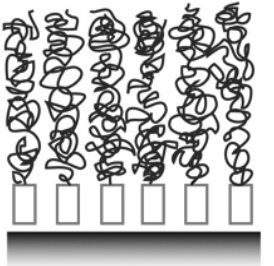

High PEG Density

Fig. 6. The ternary conjugate 16, hybridizing three different chemical structures with different functions. The resulting assembled monolayer is thought to form tightly packed brushes.

under difficult conditions [36]. Oligomers of DOPA were also successfully used to modify titanium oxide surfaces [37]. Second, the positive charge of the anachelin chromophore might contribute favorably to the binding enthalpy with the negatively charged surfaces ( $c f$. also the polycationic surface active polymers [38]). Third, the PEG chains could in principle allow for the prevention of the unwanted attachment of proteins to the surface [39]. A requirement for this would be that they could be densely packed on the surface to form stable polymer brushes.
The synthesis of the anachelin chromophore PEG conjugate was based on the biomimetic assembly of the tetrahydroquinolinium system. To achieve this goal, DOPA was converted to the precursor 11 [14][16][17]. The diamine $\mathbf{1 1}$ served as substrate for the biomimetic oxidative aza annulation reaction. Treatment of $\mathbf{1 1}$ with a tellurium oxide resulted in the clean formation of the tetrahydroquinolinium system 12 (Scheme 1).

As such, tellurium oxides should not be considered as biomimetic, as this cyclization probably involves enzymatic catalysis. Such a candidate could originate from the family of catechol oxidases. These enzymes oxidize catechols to quinones and are involved in many biochemical processes such as melanin (skin pigment) formation. We were pleased to find that treatment of the diamine 11 with tyrosinase resulted in the clean formation of the anachelin chromophore 12 [14]. As tyrosinase can be extracted directly from mushrooms and this procedure is applicable on a preparative scale, this route opens a favorable biotechnological preparation of this key compound (Scheme 2). Deprotection of the Boc group and conjugation to commercially available PEG-5000 active ester gave the hybrid 10, which was purified by size exclusion chromatography [35].

The anachelin chromophore PEG chimera is then dissolved under dilute conditions $(1 \mathrm{mg} / \mathrm{ml})$ and the hybridization with the $\mathrm{TiO}_{2}$ surface is carried out under cloud point conditions by a simple dip and rinse procedure in $4 \mathrm{~h}$ [35]. The resulting adlayers of $\mathbf{1 0}$ were characterized by various techniques such as variable angle spectroscopic ellipsometry, X-ray photoelectron spectroscopy and optical waveguide lightmode spectroscopy [35]. All these data support the presence of monomolecular self-assembled layers on the titanium oxide surface. It is reasonable to assume that there is covalent attachment of these compounds on $\mathrm{TiO}_{2}$, based on literature precedence of related systems [37].

These resulting adlayers were also characterized from the protein resistance properties, i.e. whether the modified surfaces will be resistant to the attachment of proteins from serum. This so-called biofouling is a serious problem in medicine, and a large part of research in biomedical engineering is devoted to the generation of such protein resistant surfaces. Interestingly, the surfaces coated with the anachelin chromophore PEG conjugate were highly resistant towards the attachment of proteins from serum. This correlates in general well with a dense packing of the polymer brushes on the surface (Fig. 6). Control experiments involving different linkers such as dopamine, minosine and DOPA all resulted in lower adlayer thickness and therefore a dramatic loss of protein resistance [35].

The hybrid 13 can be considered to possess ternary structure, as three different chemical structures $\left(\mathrm{TiO}_{2}\right.$, anachelin chromophore, and PEG) with different functional properties (solid support, binding and protein resistance) are combined. This exemplifies the power of natural product hybrids as fundamentally different properties can be combined to generate innovative solutions for challenges in medicine. 


\section{Conclusion}

This highlight article gives an overview over different classes of natural product hybrids. Structural natural product hybrids can be considered as compounds in which fragments of different biosynthetic origin are combined. This is exemplified by the cyanobacterial siderophore anachelin, which is composed of alkaloid, unnatural peptide and polyketide fragments. A second class is composed of functional natural product hybrids, where substructures with different biological functions are combined. As an example, the hybrid between nostocarboline and ciprofloxacin was discussed, which delivers an unprecedented broad spectrum of biological activity. Last, higher order hybrids were introduced by the ternary compound $\mathrm{TiO}_{2}$-anachelin chromophore and PEG. This example is interesting since inorganic metal oxides are hybridized to a natural product and a synthetic polymer to generate protein-resistant surfaces. This ternary hybrid also shows the possibilities in designing and tailoring more complex hybrids that can be used to suit a well-defined molecular function.

\section{Acknowledgments}

I warmly thank my coworkers, past and present, in particular Dr. Yann Bethuel, Barbora Malisova, Damien Barbaras and Tobias Brütsch for their great experimental and intellectual efforts. In addition, such transdisciplinary research would not be possible without my excellent collaborators in limnology at the University of Zurich, Dr. Judith Blom and Prof. Dr. Friedrich Jüttner, and in materials science at ETH Zürich, Prof. Dr. Marcus Textor. I am also grateful to Dr. Stefan Zürcher and Dr. Samuele Tosatti of SurfaceSolutionS GmbH and Dr. Hans Locher and Dr. Christian Hubschwerlen of Actelion AG for wonderful collaborations. Financial support from Prof. Dr. Erick M Carreira in the context of my habilitation is gratefully acknowledged as is support from the Roche Research Foundation, Swiss National Science Foundation, ETH Zürich, and the Latsis Foundation.

Received: October 31, 2006

[1] Reviews: M.S. Butler, J. Nat. Prod. 2004, 67, 2141-2153; G.A. Cordell, Phytochemistry 2000, 55, 463-480.

[2] G.M. Cragg, D.J. Newman, K.M. Snader, J. Nat. Prod. 1997, 60, 52-60.

[3] D.J. Newman, G.M. Cragg, K.M. Snader, J. Nat. Prod. 2003, 66, 1022-1037.

[4] L.F. Tietze, H.P. Bell, S. Chandrasekhar, Angew. Chem. Int. Ed. 2003, 42, 3996-4028; C. Chen, X. Li, C.S. Neumann, M.M.-C. Lo, S.L. Schreiber, Angew. Chem. Int. Ed. 2005, 44, 2249-2252. A. Ganesan, Curr. Opin. Biotechnol. 2004, 15, 584-590.

[5] See for example the work of Waldmann and coworkers: L. Brunsveld, J. Kuhlmann, H. Waldmann, Methods 2006, 40, 151-165.
[6] Reviews: W. Keller-Schierlein, V. Prelog, H. Zähner, Fort. Chem. Org. Nat. 1964, 22, 279-322; K.N. Raymond, G. Müller, B.F. Matzanke, Top. Curr. Chem. 1984, 123, 49-102; H. Drechsel, G. Jung, J. Pept. Sci. 1998, 4, 147-181.

[7] R. Crichton, 'Inorganic Biochemistry of Iron Metabolism: From Molecular Mechanism to Clinical Consequences', 2nd. Ed., John Wiley \& Sons, New York, 2001.

[8] N.M. Price, F.M.M. Morel, Metal Ions Biol. Syst. 1998, 35, 1-36; S.W. Wilhelm, D.P. Mazwell, C.G. Trick, Limnol. Oceanogr. 1996, 41, 89-97; S.W. Wilhelm, K. MacAuley, C.G. Trick, Limnol. Oceanogr. 1998, 43, 992-997.

[9] S.J. Goldman, P.J. Lammers, M.S. Berman, J. Sanders-Loehr, J. Bacteriol. 1983 156, 1144-1150; P.J. Lammers, J. SandersLoehr, J. Bacteriol. 1982, 151, 288-294; F.B. Simpson, J.B. Neilands, J. Phycol. 1976, 12, 44-48.

[10] Y. Ito, A. Butler, Limnol. Oceanogr. 2005, 50, 1918-1923.

[11] H. Beiderbeck, K. Taraz, H. Budzikiewicz, A.E. Walsby, Z. Naturforsch. 2000 55c, 681-687; H. Beiderbeck, Dissertation, Universität zu Köln, Dr. Dirk Blunk Verlag, 2000. Anachelin was probably isolated 30 years ago, but its structure was not characterized: A.E. Walsby, Br. Phycol. J. 1974, 9, 383-391

[12] Y. Itou, S. Okada, M. Murakami, Tetrahedron 2001, 57, 9093-9099; Y. Ito, K. Ishida, S. Okada, M. Murakami, Tetrahedron 2004, 60, 9075-9080.

[13] K. Gademann, H. Budzikiewicz, Chimia 2004, 58, 212-214.

[14] K. Gademann, ChemBioChem 2005, 6, 913-919.

[15] Y. Bethuel, K. Gademann, J. Org. Chem. 2005, 70, 6258-6264.

[16] K. Gademann, Y. Bethuel, Angew. Chem. 2004, 116, 3389-3391; Angew. Chem. Int. Ed. 2004, 43, 3327-3329.

[17] K. Gademann, Y. Bethuel, Org. Lett. 2004 6, 4707-4710.

[18] Reviews: S. Singh, B.N. Kate, U.C. Banerjee, Crit. Rev. Biotech. 2005, 25 , 73-95; A.M. Burja, B. Banaigs, E. AbouMansour, J.G. Burgess, P.C. Wright, Tetrahedron 2001, 57, 9347-9377.

[19] Isolation: L. Hagmann, F. Jüttner, Tetrahedron Lett. 1996, 37, 6539-6542; Synthesis: U. Papke, E.M. Gross, W. Francke, Tetrahedron Lett. 1997, 38, 379-382.

[20] See, for example: J.F. Blom, B. Bister, D. Bischoff, G. Nicholson, G. Jung, R.D. Süssmuth, F. Jüttner, J. Nat. Prod. 2003 , 66, 431-434.

[21] K. Stratmann, R.E. Moore, R. Bonjouklian, J.B. Deeter, G.M.L. Patterson, S. Shaffer, C.D. Smith, T.A. Smitka, J. Am. Chem. Soc. 1994, 116, 9935-9942.

[22] L.K. Larsen, R.E. Moore, G.M.L. Patterson, J. Nat. Prod. 1994, 57, 419-421.

[23] R.B. Volk, J. Appl. Phycol. 2005, 17, 339347; R.B. Volk, J. Appl. Phycol. 2006, 18, 145-151.
[24] S. Kodani, A. Imoto, A. Mitsutani, M. Murakami, J. Appl. Phycol. 2002, 14, 109-114.

[25] P.G. Becher, J. Beuchat, K. Gademann, F. Jüttner, J. Nat. Prod. 2005, 68, 1793 1795.

[26] E. Flores, C.P. Wolk, Arch. Microbiol. 1986, 145, 215-219.

[27] Van Tamelen et al. proposed to have isolated the $\mathrm{C}-6 \mathrm{Cl}$ species, but did not provide sufficient characterization: E.E. Van Tamelen, V.B. Haarstad, R.L. Orvis, Tetrahedron 1968, 24, 687-704.

[28] J.W. Armit, R. Robinson, J. Chem. Soc. 1925, 127, 1604-1618; A.P. Gray, J. Am. Chem. Soc. 1955, 77, 5930-5932.

[29] Review: A. Nordberg, A.-L. Svensson, Drug Safety 1998, 19, 465-480. For an overview of carboline alkaloids in PD treatment, see: T.L. Sourkes, Can. J. Physiol. Pharmacol. 1999, 77, 375-382.

[30] J.F. Blom, T. Brütsch, D. Barbaras, Y. Bethuel, H.H. Locher, C. Hubschwerlen, K. Gademann, Org. Lett. 2006, 8, 737740.

[31] Harmane and norharmane were reported to have algicidal properties, see references [23][24].

[32] C. Hubschwerlen, J.-L. Specklin, C. Sigwalt, S. Schroeder, H.H. Locher, Bioorg. Med. Chem. 2003, 11, 23132319.

[33] Reviews: A.T. Stone, Rev. Mineralogy 1997, 35, 309-344; S.M. Kraemer, Aquatic Sci. 2004, 66, 3-18.

[34] M.J. McWhirter, P.J. Bremer, I.L. Lamont, A.J. McQuillan, Langmuir 2003 , 19, 3575-3577; S.-Y. Kang, P.J. Bremer, K.-W. Kim, A.J. McQuillan, Langmuir 2006, 22, 286-291.

[35] S. Zürcher, D. Wäckerlin, Y. Bethuel, B. Malisova, M. Textor, S. Tosatti, K. Gademann, J. Am. Chem. Soc. 2006, 128, 1064-1065.

[36] J.H. Waite, M.L. Tanzer, Science 1981, $212,1038-1040$

[37] J.L. Dalsin, B.-H. Hu, B.P. Lee, P.B. Messersmith, J. Am. Chem. Soc. 2003, 125 , 4253-4258; J.L. Dalsin, L. Lin, P.B. Messersmith, S. Tosatti, J. Vörös, M. Textor, Langmuir 2005, 21, 640-646; A.R. Statz, P.B. Messersmith, R.J. Meagher, A.E. Barron, J. Am. Chem. Soc. 2005, 127, 7972-7973; M.J. Sever, J.T. Weisser, J. Monahan, S. Srinivasan, J.J. Wilker, Angew. Chem. Int. Ed. 2004, 43, 448-450.

[38] See, for example, S. Pasche, S.M. De Paul, J. Vörös, N.D. Spencer, M. Textor, Langmuir 2003, 19, 9216-9225

[39] See, for example, J.H. Lee, J. Kopecek, J.D. Andrade, J. Biomed. Mat. Res. 1989 , 23, 351-368; K.L. Prime, G.M. Whitesides, Science 1991, 252, 1164-1167; N.P. Desai, J.A. Hubbell, Biomaterials 1991, 12, 144-153. 